\title{
Coping with Stress of the Military Men in a Period of Organizational Changes
}

\author{
Polina Semyanishcheva \\ Faculty of psychology, Department of Work and \\ Engineering Psychology \\ Lomonosov Moscow State University \\ Moscow, Russia
}

\author{
Zoya Dudchenko \\ Faculty of psychology, Department \\ of psychological support of professional activities \\ Saint Petersburg State University \\ Saint Petersburg, Russia
}

\begin{abstract}
The present study aimed at investigating the subjective perceptions of the officers about the professional stress factors and features of coping with them. Work has been done on the contingent of military men in the period of the reforms in the army. It was assumed that, in a period of change, features of coping behavior of military personnel would be especially bright because the tension labor situation will require the activation of individual resources that contribute to the successful overcoming of novelty and uncertainty. To identify striking features of coping in stressful situations the indicators of coping are considered in connection with the level of job satisfaction. The study revealed the following peculiarity of military men's coping with difficult situations: the use of problem-oriented coping strategy and the use of avoidant, emotional-oriented strategies, impulsive and aggressive actions.
\end{abstract}

Keywords-stress; functional state; coping behavior; job satisfaction; innovations; military men

\section{INTRODUCTION}

In modern times, the constant companion of the development of all branches are continuous and accelerating changes, which represent large-scale sectoral reforms, aimed at the modernization of labor and to improve the efficiency of organizations $[1,5]$. In the period of reform in various sectors, scientists and experts pay great attention to organizational problems and the development of resistance to stress of employees.

The problem of innovation in organizations takes an important place in the modern organizational psychology and psychology of professional health. Dramatic changes in human life are important; even positive change can lead stress. At all stages of innovative changes, psychological problems in the organization are inevitable. People mostly negatively perceived change and this is quite normal because they need to adapt to them $[4,5,7]$.

Organizational stress factors along with professional stressors have a negative impact on the health of employees, their job satisfaction and effectiveness. In general, stress is the main source of negative impact on the well-being of employees among different types of adverse functional states $^{1}[2,4,8]$.

During a period of stress, employees use coping behaviors that support their psychosocial adaptation. These techniques and approaches aimed at correcting the conditions that lead to stress whose strength is determined not so much by the quality of the stressor but more so by the significance of the situation for the human. The spectrum of variants of coping behavior is very wide - from avoidance to qualitative transformation of a situation or personality. Some of them have the most positive impact on the human condition, others negative $[4,6,7,8]$.

In the analysis of the characteristics and success of coping behavior is an important parameter of job satisfaction. It is important to clarify that job satisfaction may be considered as a factor determining the peculiarities of coping and, as a result, the effectiveness of self-regulation condition. So if the employee is aimed at the effective implementation of activities, it leads to more effective processes of selfregulation and, as a result, higher job satisfaction overall. Job satisfaction, in turn, affects the attitude of employees to work and, therefore, the motivation to further activity. It is understood as a construct, a synthesis of the subjective vision of the person of all components of the labor process in particular (including the organizational) conditions for its implementation taking into account the balance between the level of aspiration of the employee and their implementation. Note that job satisfaction is one of the key criteria for successful adaptation to innovation process $[4,8,9]$.

The successful application of methods and means of selfregulation of functional states that an adequate situational requirements, increases resistance to stress of its staff, supports efficiency and contributes to the subjective balance of human capabilities and requirements in a busy and innovative period.

\section{The AIM AND Methods OF RESEARCH}

Changes, often held in large state institutions, did not pass by such conservative structure as the army. Recently,

1 Functional status of a person - is an integral complex of the functions and qualities that directly or indirectly cause the execution of working activities [8] 
the Russian Armed Forces carried out large-scale reform: changes have been made to the structure of military units, the motivational system of employees and much more.

A general overview of the work military personnel's nature reveals a high degree of extremeness and stress level regarding this profession and the importance of stability of mental state for military performance and their own health. Therefore, it is necessary to provide psychological training and military assistance that would prepare them for coping with difficult life situations both directly and indirectly with the service.

The aim of the study was to identify the peculiarities of coping behavior of officers under innovation stress. The study focused on the subjective view of stress factors and coping strategies during the reform of the military sector. The study involved 130 officers aged 20 to 42 years.

Given the large-scale reforms, the army expected that it will be possible to identify the distinct features of coping behavior of officers because the stress of the situation will require increased involvement of individual behavioral resources, contributing to successfully overcome the situation changes. Of course, professional's proactive position, innovative readiness and a use of productive behavioral models should lead to more successful adaptation to the conditions of the innovative stress $[2,4,8]$.

The methods included the following questionnaires:

\section{A. Job stress survey (JSS) (Ch. Spielberger, adapted by A. Leonova) [4].}

In the questionnaire, there is a list of 30 possible sources of stress in professional activities (e.g., "hard deadlines") for which it is necessary to estimate the frequency and force of impact by a rising 9-point scale (where 5 points is a "normal" level of stress inherent in any job). In addition to the amount and the average strength and frequency of impact of stress factors for each item, their weight is calculated by multiplying the values of force and frequency. The method was chosen since to detect a large number of specific stressors in professional and organizational environment.

\section{B. Job satisfaction questionnaire (V. Rozanova) [1].}

The questionnaire contains 14 statements (e.g., "your satisfaction with the teamwork of employees"), each of which need to evaluate, choosing one of the 5 response options rating scale from 1 to 5 points ( 1 point - "quite satisfied", 5 points - "extremely unsatisfied"). At the end of the questionnaire, scores are summed into a single index. The questionnaire was used to explore employee satisfaction with working conditions, content of activities and relationships with colleagues.

\section{Coping inventory for stressful situations (CISS) (N. Endler, J. Parker, adapted by T. Krukova) [3].}

The questionnaire aimed to identify the dominant types of coping, measuring three basic styles of coping behavior: problem-focused coping, emotional-focused coping and coping focused on avoidance (and 2 additional scales - distraction and social diversion). In the questionnaire, there is a list of the 48 possible human reactions to various challenges (e.g., "I try to be in crowded places"), the frequency of which you need to evaluate on a 5-point scale, where a score of 1 is never and 5 points - very often. The technique was used to identify common strategies of coping of the officers with a stressful situation.

\section{The strategic approach to coping scales questionnaire (SACS) (S. Hobfoll, adapted by N. Vodopyanova \& E. Starchenkova) [2].}

The questionnaire is built based on the multi-axial model of coping behavior and involves the identification 9 models of behavior under stress. Subjects had to rate on a 5-point scale ( 1 = "no, this is not true", 5 - "absolutely") features their typical behavior in various stress situations that are offered in 54 claims (e.g., "in any difficult situations you don't give up"). The results include 9 indicators of assertive behavior, introduction in social contact search of social support, avoidance, cautious, impulsive, manipulative, antisocial and aggressive actions. The methodology was included in the diagnostic package with the aim of identifying specific patterns of coping behavior officers. Thus, the last two techniques have allowed identifying the characteristics of coping related to different levels of implementation of coping behavior in a stressful situation.

The obtained data were processed using the statistical package SPSS 16.0 for Windows. Processing methods included descriptive statistics, correlation analysis, methods of comparisons of samples and others.

The results of the study showed that the most severe stress factors in the work of the employees are working overtime $(\mathrm{M}=37.07 / \sigma=21.309)$, limited opportunities for professional growth $(\mathrm{M}=33.26 / \sigma=19.860)$, a contradiction assigned tasks and responsibilities $(\mathrm{M}=34.33 / \sigma=20.797)$, unfairness in pay $(M=34.27 / \sigma=23.539)$, excessive burden of work with documentation and help information $(M=31.76 / \sigma=20.618)$, lack of time to meet personal needs and leisure $(\mathrm{M}=32.60 / \sigma=21.587)$, the need to perform work for others - colleagues, bosses, subordinates $(\mathrm{M}=35.87 / \sigma=22.108)$. All other factors fall into the range of moderate stress.

The least stressful for officers were: conflicts with other parts of the organization $(M=17.77 / \sigma=14.042)$, insults and abuse from the customer/consumer/client/partner $(\mathrm{M}=18.03 / \sigma=14.024)$, lack of participation in planning and making organizational decisions $(M=19.94 / \sigma=13.491)$, periods of forced inactivity $(M=18.83 / \sigma=14.150)$.

Job satisfaction of the respondents showed medium level $(\mathrm{M}=38.64 / \sigma=9.083)$. The most strongly linked to the levels of stress factors overtime $(\mathrm{r}=0.491, \mathrm{p}<0.001)$ and limited opportunities for professional growth $(\mathrm{r}=0.489, \mathrm{p}<0.001)$.

Thus, the study of subjective assessment of stress professional activities revealed a number of potential reasons for the formation of negative functional states of the officers. The respondents have stress in different areas of working life, for example, professional growth and the satisfaction of 
personal needs. It should be noted that the severity of the impact of the main stressors is formed mainly by subjective frequent exposure, and not by the subjective importance of specific stress factor.

That is, the negative aspects of working life are not strong enough to force them to abandon activity but frequent enough to provide the ground for the emergence of adverse functional state and of job dissatisfaction among professionals.

Study of self-regulation of stress included identifying strategies of coping (according to the results of the questionnaire of coping inventory for stressful situations (CISS) and models of coping (according to the results of the strategic approach to coping scales questionnaire (SACS).

In the first case, the results showed the following:

- problem-oriented coping has an average intensity $(57,38 / \sigma=8,195)$,

- emotional-focused coping has a low intensity $(33,47 / \sigma=9,527)$,

- coping focused on avoidance has a high intensity $(43,45 / \sigma=10,615)$.

The severity of strategies of distraction and social diversion have an average intensity $(18,48 / \sigma=5,938$; $16,17 / \sigma=3,652$, respectively).

In the second case, no identified high or low performance in the use of any specific models of coping (medium level of severity on all scales): assertive action $-M=20.30 / \sigma=3.350$, entry into social contact $-M=22.73 / \sigma=3.965$, search for social support $-\mathrm{M}=22.52 / \sigma=4.599$, careful steps $-\mathrm{M}=\mathrm{of}$ $20.53 / \sigma=3.896$, impulsive actions $-M=17.33 / \sigma=3.349$, avoidance - $\mathrm{M}=15.79 / \sigma=3.608$, manipulative action $\mathrm{M}=19.64 / \sigma=4.143$, antisocial actions - $\mathrm{M}=17.82 / \sigma=4.672$, aggressive actions $-\mathrm{M}=16.82 / \sigma=5.052$.

So, in the period of industry changes, military men have an average job satisfaction. The more troops unhappy with aspects of work relating to high load and complexity of professional growth, the less satisfied they are with their work. The study of peculiarities of self-regulation of the functional state showed that, despite the high ratings of stress that is part of the aspects of professional activities, the officers have the behavioral resources to cope with difficult situations: officers do not sink too much in the emotional assessment of the event but rather tend to actively overcome that in difficult situations can improve their satisfaction.

Meanwhile, to an even greater extent, the servicemen resort to avoidant coping strategies. Likely, limited impact on innovations provokes employees to appeal to avoidant coping strategies.

To identify additional features in the actualization of specific strategies of coping were compared characteristics of coping for officers with different levels of job satisfaction. For this purpose has been allocated 2 groups of respondents: a group with a high rate of satisfaction - group $1(\mathrm{M}=29.63$ / $\sigma=4.307,46$ people, $35.7 \%$ of the sample) and group with lowest rate of satisfaction - group $2(\mathrm{M}=48.43 / \sigma=5.376,47$ people, $36.4 \%$ of the sample). The difference between groups in the level of job satisfaction are statistically significant $(\mathrm{t}=-18.582 ; \mathrm{p}<0.001)$ and similar in marital status, age and average term of service in the army.

Data processing on selected groups gave the following results. Officers of the first group, who are satisfied with the work, do not experience pronounced negative impact of aspects of employment. Despite this, the most stressful to them are the factors of overtime $(\mathrm{M}=27.65 / \sigma=19.505)$, load documentation and information $(\mathrm{M}=28.91 / \sigma=21.015)$, strict deadlines $(M=28.59 / \sigma=17.537)$, lack of time for personal needs and leisure $(M=29.26 / \sigma=19.621)$ (the range of moderate stress).

The set of factors of working stress among the officers of the second group, dissatisfied with work, is wider. In addition, subjective assessment of the significance of stress factors is higher than that of the officers of the first group, and is in the range of high stress, for example, unpleasant duty $(\mathrm{M}=34.34 / \sigma=16.629, \quad \mathrm{z}=-3.011 / \mathrm{p}=0.003)$, overtime $(\mathrm{M}=48.19 / \sigma=21.001, \quad \mathrm{z}=-4.367 / \mathrm{p}=0.000), \quad$ limited opportunities for professional growth $(M=45.60 / \sigma=20.737$, $\mathrm{z}=-5.087 / \mathrm{p}=0.000)$, lack of leadership support and others $(\mathrm{M}=40.77 / \sigma=18.488, \quad \mathrm{t}=-5.375 / \mathrm{p}=0.000)$. By comparing respondents of the second group, they are ranked higher in stressful factors such as the need to fulfill new and unfamiliar tasks $(\mathrm{M}=27.70 / \sigma=17.361, \mathrm{z}=-2.554 / \mathrm{p}=0.011)$, the need to overcome crisis situations $(\mathrm{M}=28.98 / \sigma=13.329$, $\mathrm{t}=$ $3.30 / \mathrm{p}=0.001$ ) (the range of moderate stress). That illustrates the negative perception of innovative changes.

As can be seen, the officers of the second group with low job satisfaction have higher level of stress. The major reforms with uncertain success are likely the cause of strong emotions, confusion and stress of the officers.

As for the features of coping, the officers of the first group, satisfied with the work, have a medium level of problem-focused and avoidant coping strategies $(\mathrm{M}=57,54 / \sigma=8,068, \mathrm{M}=43,04 / \sigma=10,362$ respectively) and low level of emotional-oriented coping strategy $(\mathrm{M}=31,61 / \sigma=10,325)$. The severity of strategies of distraction and social diversion also have an average intensity $(\mathrm{M}=18,17 / \sigma=5,881, \mathrm{M}=16,09 / \sigma=3,681$ respectively $)$ Also, they tend to have low level of application of the impulsive actions $(\mathrm{M}=16,98 / \sigma=3,214)$.

The level of different strategies and models for overcoming stressful situations of the officers of the second group is average. But the data comparison showed that the officers of the second group more often use emotionaloriented coping strategy $(\mathrm{M}=36,04 / \sigma=8,600, \quad \mathrm{t}=$ $2,252 / \mathrm{p}=0,027)$ and manipulative, aggressive steps $(\mathrm{M}=20,77 / \sigma=3,409, \mathrm{t}=-2,310 / \mathrm{p}=0,023$ and $\mathrm{M}=18,04 / \sigma=4,544$, $\mathrm{t}=-2,418 / \mathrm{p}=0,018$ respectively): they feel less control over events in their lives, focus on the emotional experience of the problems and exhibit a more aggressive behavior in response to stressful situations.

According to the results of comparative analysis between groups with high and low job satisfaction, we can make the 
assumption that the officers of the second group were more sensitive to the challenges of the modern situation and many reforms in the army. They are significantly characterized by the use of less effective strategies and models of coping from the standpoint of organizational norms [6].

\section{THE CONCLUSIONS OF THE WORK}

According to the research results, we can make the following conclusions:

- The study of subjective assessment by officers of the extent of the negative impact of aspects of professional and organizational environment have shown the presence of psychological tension. Among the stress factors can distinguish a high workload, poor career prospects, the experience of discrepancy between the cost of the forces and their payment and others. In terms of military reform officers, they demonstrate a sufficient level of activity and try not to take in the emotional assessment of the events. At the same time, limited possibilities of influence on innovation provoke the military personnel on an appeal to the coping strategy of avoidance as a subjectively acceptable "way out" from under the pressure of circumstances.

- It can be assumed that differences in the perception of the organizational processes of reforming the army and future prospects associated with using various resource and style of regulation of the functional state of the officers. So, officers, who feel the high degree of tension of professional situations, appeal to more aggressive and manipulative acts and emotionally fixed on a situation without a feeling of control over what is happening. The inefficient application of strategies and models of coping inadequate to requirements of the situation of innovative transformations is reflected in low job satisfaction.

- At the same time officers, who are less sensitive to the negative aspects of innovative processes in the industry have low level of emotional-oriented coping and are more satisfied with the work. In this case, productive style to overcome stressful situations of the officers can maintain a high ability to adapt to innovative stressors and a high level of job satisfaction.

In the period of reform in the army, psychological support of military personnel is particularly important because of changing regulations and working conditions, the emergence of feelings of uncertainty and insecurity in their future [1].

One of the factors of occurrence of stress may be the lack of flexible adaptation and use a variety of coping strategies appropriate to the characteristics of the situation. Develop productive coping patterns of employees is necessary, as the stress can even improve performance, if acts as the operational state of mobilization [8]. So, psychological work on optimization of activities of military personnel should include the training of a most successful and positive ways of coping behavior.

With the help of psycho-prophylactic activities and providing psychological support, the amount of psychological casualties among military personnel is reduced. In close relationship with the military, training must be carried out for the psychological aspect of servicemen because, in the presence of high-level skills of psychological self-control and high combat readiness, the activities of the officer may be subject to the will and desire to accomplish the task.

The study of coping of the military men allows understanding more the possibility of optimizing their functional states and efficiency in work. Development of directions and methods of psychological work to reduce the adverse impact of implemented changes should take into account the peculiarities of overcoming stressful situations for employees with different levels of job satisfaction. The results of the study can be used for the planning of psychological support of organizational changes in the army, psychological counseling officers in stressful situations and programs of psychological training of officers.

\section{REFERENCES}

[1] I.S. Brazevich, "Innovations as a factor of successful adaptation of the company to environment changes", Dialogue of cultures - 2010: Science in knowledge society: collection of scientific papers of International scientific-practical conference. St. Petersburg: Publishers of St. Petersburg Academy of Management and Economics, 2010. -424 p.

[2] Z.F.Dudchenko, "Ensuring a healthy lifestyle at the state level".Scientific notes SPbGIPSR.-№1 (15).-2011.-C.87-91

[3] L. A. Vereshchagina, "Evaluation of job satisfaction" // Workshop on psychology of management (ed. by G. S. Nikiforov, M. A. Dmitrieva, V. M. Snetkov). SPb.: Peter, 2003. P. 311-321.

[4] N. Vodopyanova, E. Starchenkova, Psychodiagnostics of stress. St. Petersburg: Peter, 2009. - 336 p.

[5] T. S. Kabachenko, Psychology in human resource management: textbook. - St. Petersburg: Peter, 2003. - 400 p.

[6] T. L. Krukova, Methods of studying coping: three coping scale. Kostroma: Avantitle, 2007. - 60 p.

[7] R.S. Lazarus, S. Folkman. Stress, appraisal and coping. N.Y.: Springer, 1984. - $218 \mathrm{p}$

[8] A. B. Leonova, S. B. Velichkovsky, "Differential diagnosis conditions reduced performance", The Psychology of mental States (ed. by O. A. Prokhorov). Kazan: Center for innovative technology, 2002. Vol. 4. p. $326-343$.

[9] P. A. Semyanishcheva, Psychological resources of coping with stress in the situation of the industry organizational changes (the military contingent), Moscow University Physics Bulletin, Series 14 Psychology. No. 1, 2013. - p. 123 - 135. 